\title{
Utilização de valvas homólogas e heterólogas em condutos extracardíacos
}

\author{
Rui Siqueira de ALMEIDA*, Richard WYSE**, Marc DE LEVAL ${ }^{\star \star \star}$, Jaroslav STARK ${ }^{\star \star \star}$
}

\begin{abstract}
ALMEIDA, R. S.; WYSE, R.; DE LEVAL, M.; STARK, J. - Utilização de valvas homólogas e heterólogas em condutos extracardiacos. Rev. Bras. Cir. Cardiovasc., 3(2): 101-108, 1988.

RESUMO: O conceito do uso de um conduto extracardíaco para estabelecer uma via de saída, conectando o ventrículo direito com o tronco pulmonar, ou seus ramos, foi desenvolvido na década de 60. Entre 1971 e 1986, 335 pacientes receberam, no The Hospital for Sick Children, de Londres, condutos extracardiacos para o lado direito do coração; 176 destes foram homoenxertos aórticos, preservados em solução antibióticonutriente; 140 heteroenxertos (Hancock, Ross, Carpentier-Edwards, lonescu-Shiley e 19 tubos não valvulados. Estes condutos foram usados na correçāo de defeitos cardiacos complexos. A idade média foi de 6,34 anos e o peso médio, de $17,8 \mathrm{~kg}$. O diâmetro interno dos condutos variou de 8 a $30 \mathrm{~mm}$. A mortalidade hospitalar foi de $29,2 \%$ e o seguimento dos sobrevivente teve uma duração máxima de 14,3 anos, sendo que apenas $40 \%$ delas foram relacionadas ao conduto extracardiaco. A curva atuarial, livre de obstrução, dos condutos extracardiacos foi significativa, quando se analisaram os homoenxertos, face a cada grupo de heteroenxertos $(p<0,005)$. Os fatores de risco mais importantes foram: o número de complicaçōes pós-operatórias, para óbitos tardios; a severidade das lesões associadas, para as reoperaçōes; a data da cirurgia, para a troca do conduto extracardiaco, e a severidade das lesōes associadas, para a obstrução. Assim, conclui-se que, a longo prazo, o uso de condutos valvulados extracardiacos tem bons resultados, especialmente no que se refere aos homoenxertos. Pelos dados apresentados, os homoenxertos aórticos, preservados em solução antibiótico-nutriente, continuam a ser nossa primeira escolha na reconstrução da via de saída ventrículo-pulmonar.
\end{abstract}

DESCRITORES: condutos extracardiacos, cirurgia; valvas cardiacas, cirurgia; próteses valvulares cardiacas, cirurgia.

\section{INTRODUÇĀO}

O conceito do uso de condutos extracardiacos (CE) na correção de defeitos cardíacos congêntos, caracterizados pela descontinuidade entre o ventrículo direito, ou átrio direito, e o tronco pulmonar, ou os seus ramos, foi estabelecido com o trabalho experimental de ROBBARD \& WAGNER ${ }^{20}$, em 1949. Porém, somente na década de 60 iniciaram-se as primeiras cirurgias, quando
RASTELLI et alii ${ }^{19}$ confeccionaram um conduto não valvulado de pericárdio, para estabelecer a continuidade entre o ventrículo direito e o tronco pulmonar, numa forma complexa de tétrade de Fallot; ROSS \& SOMERVILLE ${ }^{21}$, 2 anos após, descreveram o primeiro uso de homoenxertos aórticos na correção total da atresia pulmonar com defeito do septo ventricular.

Desde então, o uso de CE tem-se popularizado e várias são as instituições ${ }^{4.7}$ 7. 8. 10. 16 que apresentam

Trabalho realizado no Hospital for Sick Children. Londres, Inglaterra.

Apresentado ao 15: Congresso Nacional de Cirurgia Cardiaca. Rio de Janeiro, RJ, 7 e 8 de abril, 1988.

- Do Hospital Evangélico de Curitiba, PR, Brasil. Senior House Officer e Research Fellow da Thoracic Unit do The Hospital for Sick Children, quando da elaboração deste trabalho.

* Do Institute of Child Health. University of London.

... Do Hospital for Sick Children.

Endereço para separatas: Rui Sequeira de Almeida. Rua Augusto Stellfeld, 2088. 80000 Curitiba, PR, Brasil. 
ALMEIDA, R. S.; WYSE, R.; DELEVAL, M.; STARK, J. - Utilização de valvas homólogas e heterólogas em condutos extracardiacos. Rev. Bras. Cir. Cardiovasc., 3(2): 101-108, 1988.

elevado número de pacientes operados, portadores dos mais variados tipos de cardiopatias congênitas.

Se, por um lado, o implante de CE veio trazer uma ajuda fundamental ao tratamento de defeitos cardiacos congênitos complexos, tornando-se possivel a sua correção total ${ }^{7,12,19,21}$, por outro lado, o número de trabalhos ${ }^{9}$. 18 que relatam complicaçōes, no seguimento a longo prazo, tem aumentado. Estes problemas incluem dificuldades técnicas ${ }^{17}$, deterioração ${ }^{22}$ e a formação de neointima $^{5}$, que obtrui os CE.

Devido ao fato de haver alternativas viáveis, quanto ao uso dos $C E^{6.13} \mathrm{e}$ à diferente evolução a longo prazo ${ }^{3 .}$ $16 .{ }^{25}$, torna-se relevante ter dados atualizados sobre 0 seu uso e o estado, a longo prazo.

No The Hospital for Sick Children, de Londres, iniciou-se o uso de CE, em 1971, com a utilização de hemoenxertos aórticos "frescos", esterelizados em meio antibiótico-nutriente. Como em outros Centros, devido a problemas na procura, também se usaram heteroenxertos, com válvulas de porco, ou pericárdio bovino. Para saber que tipo de CE tem o melhor perfil e durabilidade a longo prazo, analisa-se, neste trabalho, a experiência obtida nestes últimos.

\section{CASUÍSTICA E MÉTODOS}

Entre julho de 1971 e dezembro de 1986, 335 pacientes receberam CE, no The Hospital for Sick Children, de Londres. Foram excluidos deste estudo todos os pacientes com condutos entre o ventrículo sistêmico e a aorta. Nestes 335 pacientes, 176 receberam homoenxertos, 140 , heteroenxertos e 19 , tubos não valvulados (Tabela 1). Os homoenxertos aórticos foram preservados

\section{TABELA 1}

NÚMERO DE PACIENTES EM RELAÇÃO A CADA TIPO DE CONDUTO. NA COLUNA ENTRE PARENTESES ESTA INDICADO O NÜMERO DE PACIENTES QUE RECEBERAM ALTA HOSPITALAR.

\begin{tabular}{lrc}
\hline & & $\begin{array}{c}\text { NUMERODE } \\
\text { PACIENTES }\end{array}$ \\
\hline HOMOENXERTOS & & $176(128)$ \\
- Sem extensão & $84(58)$ & \\
- Com extensāo de Dacron & $76(58)$ & \\
- Em tubo de Dacron & $16(12)$ & \\
HETEROENXERTOS & & $140(103)$ \\
- Hancock & $97(73)$ & \\
- Ross & $25(16)$ & \\
- Carpentier-Edwards & $13(9)$ & \\
- lonescu-Shiley & $4(4)$ & \\
- Pericárdio & $1(1)$ & \\
TUBOS NĀO VALVADOS & & $19(10)$ \\
\hline
\end{tabular}

\footnotetext{
- Imuno, Sevenoaks, Kent, U.K.
}

em solução antibiótico-nutriente e conservados á temperatura de $4^{\circ} \mathrm{C}$. O processo de esterilização e estocagem foi descrito anteriormente ${ }^{2}$. O grupo dos homoenxertos foi subdividido em 3 subgrupos: sem extensão, ou somente a cúspide aórtica da valva mitral (como preconizado por SHABBO et alii ${ }^{23}$ ); a extensāo era de Dacron pré-coagulado, ou revestido com cola de Tiessel"; o enxerto era colocado em tubo de Dacron. O grupo de heteroenxertos também foi subdividido, de acordo com os diversos tipos (Hancock, Ross, Carpentier, Edwards, Ionescu-Shiley e pericárdio). A idade dos pacientes, na data da cirurgia, variou de 1 dia a 20 anos, com idade média de $6,34 \pm 4,64$ anos, e o peso variou de 63,5 $\mathrm{kg}$ (média de 17,82 $\pm 10,83 \mathrm{~kg}$ ).

Dos 335 pacientes, 157 foram submetidos a 1, 48 a 2 e 3 a 3 operaçōes paliativas prévias. O tipo de cirurgia paliativa está indicado na Tabela 2 . O diagnóstico inicial dos 335 pacientes é mostrado na Tabela 3.

Detalhes da correção intra-operatória foram determinados pelo tipo de defeito congênito, sendo que o tempo médio de circulação extracorpórea foi de 134 minutos (63-318 minutos) e o de pinçamentto aórtico, de 69 minutos (10-171 minutos). O diâmetro dos CE variou de 8 a $30 \mathrm{~mm}$, com um valor médio de $20,3 \mathrm{~mm}$.

O seguimento deste grupo de pacientes foi feito com base em informaçōes colhidas até agosto do ano de 1987, pelos cardiologistas do Serviço, ou por carta dos cardiologistas que referiram os pacientes.

Todos os dados pertinentes a cirurgias prévias, estudo hemodinâmico prévio, diagnóstico clínico, estudo hemodinâmico pós-operatório, reoperaçōes e seguimento a longo prazo foram colhidos, retrospectivamente, dos prontuários do hospital. Estes dados foram colocados num programa, especialmente feito para este fim, do Centro de Computação da Universidade de Londres e analisados pelo Pacote Estatístico de Ciências Sociais e Sistema de Análise Científica, usando Curvas de Sobrevida de Kaplan-Meier, Análise Regressional de Cox, Tabulações Cruzadas e Teste $T$, conforme apropriado. Os eventos computados foram morte, reoperação por obstrução do CE e obstrução do CE. A data de obstrução

TABELA 2

CIRURGIAS PALIATIVAS REALIZADAS EM 208 PACIENTES N:DE CIRURGIAS

SHUNTS

$$
\begin{aligned}
& \text { - Blalock-Taussig Clássico } \\
& \text { - Blalock-Hanlon } \\
& \text { - Blalock-Taussig Modificado } \\
& \text { - Waterston } \\
& \text { - Potss }
\end{aligned}
$$

BANDAGEM DO TRONCO PULMONAR

OUTROS (Ligadura de Canal Arterial. Ligadura de Colaterais, Unifocalização, etc.) 
ALMEIDA, R. S.; WYSE, R.; DELEVAL, M.; STARK, J. - Ĺtilização de valvas homólogas e heterólogas em condutos extracardiacos. Rev. Bras. Cir. Cardiovasc., 3(2): 101-108, 1988

TABELA 3

DISTRIBUICATO DAS CARDIOPATIAS CONGENITAS EM NUMMERO DE PACIENTES E MORTALIDADE HOSPITALAR E TARDIA. A PORCENTAGEM NA COLUNA DE MORTALIDADE TARDIA REFERE-SE AO TOTAL DE 237 PACIENTES

\begin{tabular}{|c|c|c|c|}
\hline \multirow{2}{*}{$\frac{\text { TIPO DE LESÄO }}{\text { Truncus Arteriosus }}$} & \multicolumn{3}{|c|}{$\begin{array}{c}\text { NUMERO DE } \quad \text { MORTALIDADE } \\
\text { PACIENTES }(\%) \text { HOSPITALAR }(\%) \text { TARDIA }(\%,\end{array}$} \\
\hline & $88(26.3)$ & $44(59)$ & $10(22,7)$ \\
\hline TGA +DSV + EP & $83(25,8)$ & $13(15,6)$ & $17(24,2)$ \\
\hline Atresia Tricúspide* & $44(13,1)$ & $11(25)$ & $5(15,1)$ \\
\hline$A P+D S V$ & $38(11,3)$ & $10(26,3)$ & $2(7.1)$ \\
\hline TCGA & $31(9,3)$ & $6(19,3)$ & $4(16)$ \\
\hline Outras"* & $51(15,2)$ & $14(27,4)$ & $4(10,8)$ \\
\hline Total & $335(100)$ & $98(29,2)$ & $42(17,7)$ \\
\hline
\end{tabular}

- inclui a hipoplasia de ventrículo direito. "inclui tétrade de Fallot com anomalia coronariana, sindrome de valva pulmonar ausente.

$A P=$ Atresia Pulmonar; $D S V=$ defeito do septo ventricular; $E P=$ estenose pulmonar; TCGA = transposiçāo corrigida das grandes artérias; $\mathrm{TGA}=$ transposição das grandes artérias.

dos CE foi impossivel caracterizar e, por esta razão, foi tomada a data do cateterismo, que indicou a obstrução, ou a data de reoperação que levou à troca do $C E$. A definição de obstruçāo de CE foi feita por BULL et alii $^{4}$.

\section{RESULTADOS}

Dos 335 pacientes que foram submetidos a implante de CE, $98(29,2 \%)$ morreram antes de receber alta hospitalar. Esta mortalidade está relacionada à complexidade das lesōes e às condiçōes pré-operatórias. Duzentos e trinta e sete pacientes receberam alta hospitalar e foram seguidos.

Cento e vinte e seis pacientes foram submetidos a estudo hemodinâmico, 1 mês a 12 anos (média 3,2 anos) após a cirurgia, como parte do seguimento pós-operatório. Foram realizadas 69 reoperaçôes, em 60 $(25,3 \%)$ pacientes, sendo que 14 delas foram no período de pós-operatório imediato. $\mathrm{Na}$ Tabela 4, estão indicadas

TABELA 4

PRINCIPAIS CAUSAS DE REOPERACÓES E SUA MORTALIDADE

\begin{tabular}{lrl}
\hline CAUSA & TOTAL & OBITOS \\
\hline Troca de conduto & 28 & 2 \\
- Obstrução Valvar & 7 & \\
- Obstrução do Conduto & 14 & \\
- Insuficiência Valvar & 4 & \\
- Infecção & 3 & 2 \\
Fechamento de DSV & 10 & \\
Colocação de Marcapasso & 2 & 4 \\
Outros & 15 & \\
\hline
\end{tabular}

DSV $=$ Defeito do septo ventricular as principais causas que levaram à realizção das reoperaçōes e à mortalidade hospitalar. A troca do CE foi a maior causa das reoperações $(50,9 \%)$, sendo que, em $38 \%$ destes casos, por obstrução do conduto, a qualquer nível. Na Figura 1, observam-se as obstruçōes do $\mathrm{CE}$, ao nivel das suturas próximais e distais de 2 pacientes com heteroenxertos. Um shunt residual importante, a nivel ventricular $(Q p / Q s>2: 1)$ esteve presente em $10(18 \%)$ pacientes, como causa principal da reoperação. Nas 17 outras reoperaçōes estão incluídas substituições da valva truncal, reparo/substituição da valva atrioventricular esquerda em transposiçāo corrigida das grandes artérias e colocação de marcapassos definitivos. Oito $(15,8 \%)$ dos pacientes reoperados morreram por causas relacionadas à cirurgia. A curva atuarial dos $\mathrm{CE}$ livres de reoperação é mostrada no Gráfico 1.

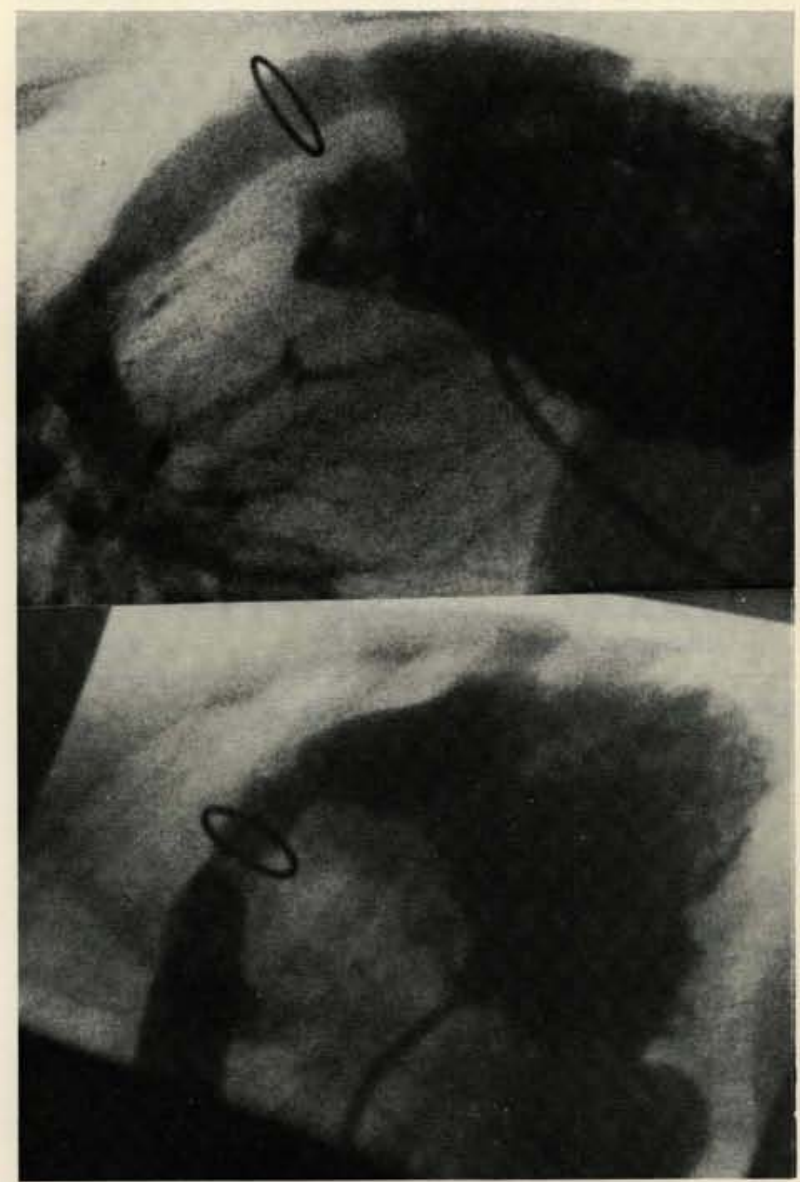

Fig. 1 - Angiogramas de pós-operatório de implante de CE. A esquer$\mathrm{da}$, estenose ao nivel da sutura proximal e, à direita, estenose ao nivel da sutura distal.

Os fatores de risco que influenciaram a sobrevida, no pós-operatório tardio, estão indicados na Tabela 5. Verificaram-se 42 óbitos tardios, cujas causas mais comuns foram infecção e insuficiência cardiaca (Tabela 6). 
ALMEIDA, R. S.; WYSE, R.; DELEVAL, M.; STARK, J. - Utilização de valvas homólogas e heterólogas em condutos extracardiacos. Rev. Bras. Cir. Cardiovasc., 3(2): 101-108, 1988

\section{GRÁFICO 1}

CURVA ATUARIAL DOS CONDUTOS EXTRACARDIACOS LIVRES DE REOPERACATO

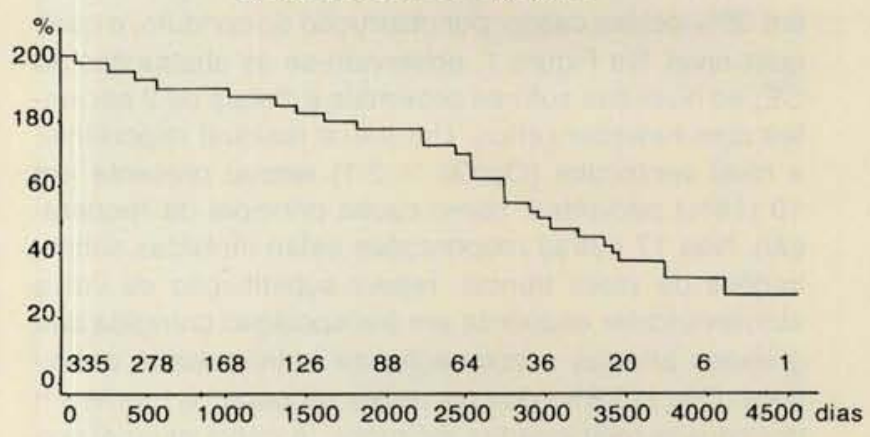

TABELA 5

FATORES DE RISCO QUE INFLUENCIARAM A SOBREVIDA NO PÓS-OPERATÓRIO TARDIO

\begin{tabular}{|c|c|}
\hline & $V A L O R D E P$ \\
\hline $\begin{array}{l}\text { 1) Fatores de risco para óbito } \\
\text { - Número de complicaçōes no pós-operatório } \\
\text { - Conduto VE para TP }\end{array}$ & $\begin{array}{l}0,0004 \\
0,0247\end{array}$ \\
\hline $\begin{array}{l}\text { 2) Fatores de risco para obstrução do CE } \\
\text { - Truncus Arteriosus } \\
\text { - Data da cirurgia } \\
\text { - Conduto de VE para TP } \\
\text { - Severidade das lesōes associadas }\end{array}$ & $\begin{array}{l}0,0049 \\
0,0172 \\
0,0291 \\
0,0447\end{array}$ \\
\hline $\begin{array}{l}\text { 3) Fatores de risco para reoperação } \\
\text { - Severidade das lesóes associadas } \\
\text { - Homoenxertos } \\
\text { - Heteroenxertos }\end{array}$ & $\begin{array}{l}0,0190 \\
0,0274 \\
0,0583\end{array}$ \\
\hline $\begin{array}{l}\text { 4) Fatores de risco para troca de CE } \\
\text { - Data da cirurgia }\end{array}$ & 0.0183 \\
\hline \multicolumn{2}{|c|}{$\begin{array}{l}\mathrm{AP}=\text { Artéria pulmonar; } \mathrm{CE}=\text { conduto extracardíaco; } \mathrm{VE}= \\
\text { ventrículo esquerdo. }\end{array}$} \\
\hline $\begin{array}{l}\text { INFECÇĀO } \\
\text { INSUFICIÊNCIA CARDÍACA } \\
\text { PÓS-REOPERAÇĀO } \\
\text { SÚBITA (ARRITMIA) } \\
\text { OUTRAS } \\
\end{array}$ & $\begin{array}{r}11 \\
10 \\
8 \\
8 \\
5 \\
\end{array}$ \\
\hline TOTAL & 42 \\
\hline
\end{tabular}

Dos 237 pacientes que sobreviveram à operação inicial, a sobrevida, ao final de 5 anos, foi de 75 e, ao final de 10 anos, de $62.5 \%$. Não houve diferença significativa nos diversos grupos de cirurgia, a não ser no grupo de atresia pulmonar, com defeito do septo ventricular, que teve melhor sobrevida (Gráfico 2).

Foram, também, comparados os grupos de homoenxertos e heteroenxertos (Tabela 7) para sobrevida, reoperação, obstrução (Gráfico 3 ) e troca do CE. Final-

\section{GRÁFICO 2}

CURVA ATUARIAL DE SOBREVIDA DOS PACIENTES QUE RECEBERAM ALTA HOSPITALAR DEPOIS DE CORRECAO DE ATRESIA PULMONAR COM DEFEITO DO SEPTO

VENTRICULAR (AP \pm DSV), ATRESIA TRICUSPPIDE OU HIPOPLASIA DE VENTRICULO DIREITO (FONTAN). TRUNCUS ARTERIOSUS E TRANSPOSICAOO DAS GRANDES ARTERIAS, DSV E ESTENOSE PULMONAR (RASTELLI)

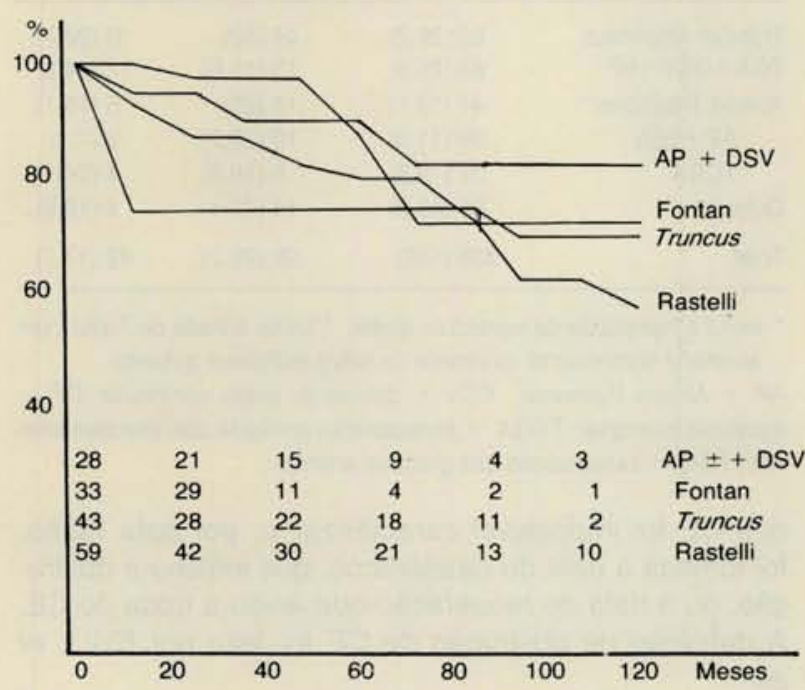

$\mathrm{AP}=$ artéria pulmonar; $\mathrm{DSV}=$ defeito do septo ventricular

TABELA 7

SOBREVIDA ATUARIAL DE HOMO E HETEROENXERTOS APÓS 5 E 10 ANOS DE SEGUIMENTO (MEDIA \pm ERRO PADRÃO)

HOMOENXERTOS HETEROENXERTOS (\%) (\%)

$\begin{array}{ccc}\begin{array}{c}\text { Sobrevida tardia } \\ 5 \text { anos }\end{array} & 77,3 \pm 5,9 & 83,5 \pm 4,0 \\ 10 \text { anos } & 53,3 \pm 13,8 & 52,8 \pm 16,3 \\ \text { Livre de reoperação } & & \\ 5 \text { anos } & 69,3 \pm 7,3 & 79,9 \pm 4,7 \\ 10 \text { anos } & 48,2 \pm 11,6 & 14,1 \pm 9,6 \\ \text { Livre de obstrução } & & \\ 5 \text { anos } & 89,3 \pm 4,3 & 80,1 \pm 5,3 \\ 10 \text { anos } & 47,0 \pm 14,2 & 26,4 \pm 15,4 \\ \text { Livre de retroca } & & \\ 5 \text { anos } & 79,9 \pm 7,2 & 92,0 \pm 3,4 \\ 10 \text { anos } & 55,9 \pm 12,2 & 23,7 \pm 15,4\end{array}$

mente, comparou-se o grupo de monoenxertos sem nenhum tipo de extensão e os CE do tipo Hancock (Tabela 8) com relação aos mesmos itens apresentados anteriormente. O Gráfico 4 mostra a curva atuarial, livre de obstrução de hemoenxertos sem estensão e CE de Hancock.

\section{DISCUSSĀO}

Desde a década de 60 , têm sido usados condutos extracardíacos no tratamento de defeitos cardíacos com- 
ALMEIDA, R. S.; WYSE, R.; DELEVAL, M.; STARK, J. - Utilização de valvas homólogas e heterólogas em condutos extracardiacos. Rev. Bras. Cir. Cardiovasc., 3(2): 101-108, 1988.

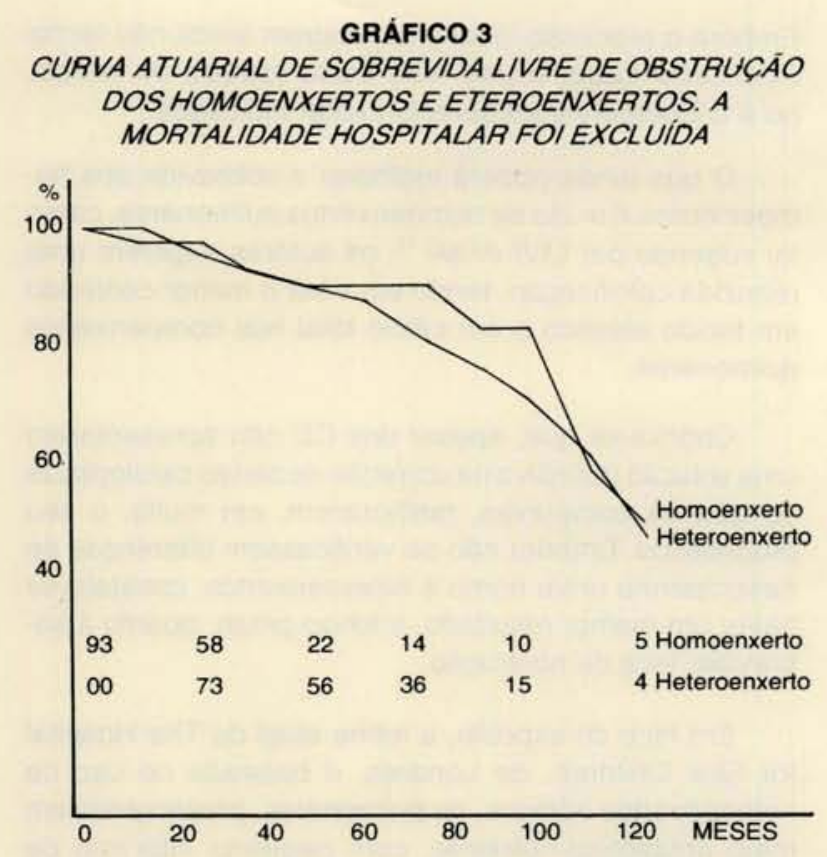

TABELA 8

SOBREVIDA ATUARIAL DE HOMOENXERTOS SEM EXTENSATO E CONDUTOS DE TIPO HANCOCK APOS 5 E 10 ANOS DE SEGUIMENTO (MEDIA \pm ERRO PADRÃO)

HOMOENXERTOS CONDUTOS SEM EXTENSÄO TIPO HANCOCK

\begin{tabular}{ccc}
\hline $\begin{array}{c}\text { Sobrevida tardia } \\
5 \text { anos }\end{array}$ & $45,9 \pm 11,5$ & $59,0 \pm 4,8$ \\
10 anos & $45,9 \pm 11,5$ & $36,8 \pm 11,7$ \\
Livre de reoperação & & \\
5 anos & $54,4 \pm 17,4$ & $84,3 \pm 4,6$ \\
10 anos & $54,4 \pm 17,4$ & $14,3 \pm 9,8$ \\
Livre de obstrução & & \\
5 anos & $93,1 \pm 4,7$ & $85,8 \pm 5,0$ \\
10 anos & $45,5 \pm 33,0$ & $28,8 \pm 15,1$ \\
Luvre de retroca & & \\
5 anos & $72,2 \pm 16,7$ & $94,5 \pm 3,1$ \\
10 anos & $72,2 \pm 16,7$ & $25,3 \pm 16,3$ \\
\hline
\end{tabular}

plexos e vários autores ${ }^{8}, 10,11,16.17$ têm apresentado seus resultados a longo prazo. No entanto, a maioria dos resultados mencionados na literatura difere dos resultados aqui relatados, não só por apresentarem um maior número de heteroenxertos, como acontece com os trabalhos americanos $5,10,15,24$, como também com os trabalhos apresentados por KAY \& ROSS " , que mostram uma exclusividade de homoenxertos. FONTAN et alii $^{8}$ mostram o uso de homoenxertos em 93 pacientes, mas não existe uma representação tão vasta de defeitos cardíacos corrigidos, pois a grande maioria são conexões atriopulmonares. Devido a estes fatos, foi feito o levantamento dos dados existentes no The Hospital for Sick Children, de Londres, nos últimos 15 anos, tendo sido

\section{GRÁFICO 4}

CURVA ATUARIAL DE SOBREVIDA LIVRE DE OBSTRUCĀO DOS HOMOENXERTOS SEM EXTENSÁO E DOS CONDUTOS TIPO HANCOCK. A MORTALIDADE HOSPITALAR FOI EXCLUIDDA

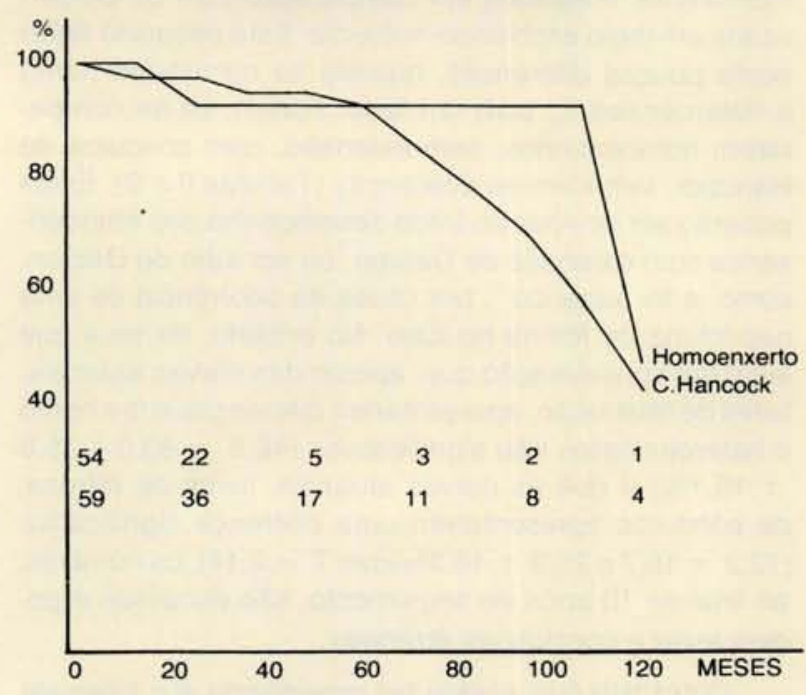

constatado que foram usados tanto homo, como heteroenxertos, pois, apesar da seleção do tipo de CE não ter sido casualizada, foram observados diversos tipos de $\mathrm{CE}$, distribuídos entre as diversas lesões corrigidas e diversas idades, devido, principalmente, ao fato de não haver disponiveis homoenxertos com diâmetro necessário, na data da cirurgia. A mortalidade hospitalar observada foi de $29,2 \%$, que pode ser considerada alta, mas que é devida à complexidade de certos defeitos cardiacos congênitos, pelo que não pode ser visto como um fator desfavorável. A complexidade na correção dos defeitos é demonstrada pelos longos períodos de circulação extracorpórea, cuja média foi de 134 minutos, na severidade das lesōes associadas, no número de complicaçōes pós-operatórias e no de cirurgias paliativas (208 em 157 pacientes).

Dos pacientes que tiveram alta hospitalar, verificouse uma sobrevida de $62,5 \%$, após 10 anos, demonstrando que este tipo de cirurgia não é uma solução eficaz, mas que melhora, indiscutivelmente, a duração e a qualidade de vida destes pacientes, mesmo levando em consideração que $12 \%$ deles necessitaram da troca do $C E$, por causas relacionadas ao mesmo, com uma mortalidade de apenas $7 \%$.

Qual o tipo de CE a ser utilizado? Será que os homoenxertos a longo prazo têm melhor desempenho que os heteroenxertos? Uma das maiores experiências com o uso de CE é a da Mayo Clinic. McGOON et alii ${ }^{15}$ publicaram 333 casos de pacientes com condutos de Hancock, 130 como homoenxertos irradiados e $5 \mathrm{com}$ condutos não valvulados. A sobrevida, ao final de 5 e 10 anos, livre de reoperação, difere da apresentada neste trabalho, tanto para os homo como para os heteroenxertos. Os fatores que podem ter contribuido para esta 
ALMEIDA, R. S.; WYSE, R.; DELEVAL, M.; STARK, J. - Utilização de valvas homólogas e heterólogas em condutos extracardiacos. Rev. Bras. Cir. Cardiovasc., 3(2): 101-108, 1988.

diferença foram levantados por STEWART et alii ${ }^{24}$, ao demonstrarem a grande incidência de obstruçâo de condutos de Hancock em pacientes assintomáticos, bem como a diferença de evolução, a longo prazo, dos homoenxertos irradiados em comparaçāo com os preservados em meio antibiótico-nutriente. Esta pesquisa apresenta poucas diferenças, quando se comparam homo e heteroenxertos, com um todo. Porém, se se compararem homoenxertos, sem extensão, com condutos de Hancock, verificam-se diferenças (Tabelas 8 e 9). Estas poderão ser devidas ao fraco desempenho dos homoenxertos com extensão de Dacron, ou em tubo de Dacron, como ja foi sugerido ${ }^{\prime}$, por causa da ocorrência de uma neo-íntima de fibrina no tubo. No entanto, ter-se-à que levar em consideração que, apesar das curvas atuariais, livres de obstruçāo, apresentarem diferenças entre homo e heteroenxertos não significativas $(46,5 \pm 33,0$ e 25,8 $\pm 15,1 \%$ ) e que as curvas atuariais, livres de retroca, de condutos apresentarem uma diferença significativa $(72,2 \pm 16,7$ e $25,3 \pm 16,3 \%$ com $T=2,14)$, os números, ao final de 10 anos de seguimento, são escassos e podem levar a conclusōes errôneas.

Outro fato que parece ter importância é o intervalo de tempo entre a dissecção e o uso de homoenxertos ${ }^{2}$.
Embora o processo ideal e estocagem ainda não tenha sido encontrado, o uso num curto espaço de tempo, ou a criopreservaçāo parecem estar indicados.

O que ainda poderá melhorar a sobrevida dos homoenxertos é o uso de homoenxertos pulmonares, como foi sugerido por LIVI et alii ${ }^{14}$; os autores sugerem uma reduzida calcificação, tendo em vista o menor conteúdo em tecido elástico e em cálcio total nos homoenxertos pulmonares.

Conclui-se que, apesar dos CE não apresentarem uma solução definitiva na correção de certas cardiopatias congênitas complexas, melhoraram, em muito, o seu prognóstico. Embora não se verificassem diferenças de desempenho entre homo e heteroenxertos, constata-se haver um melhor resultado, a longo prazo, quanto à sobrevida, livre de obstrução.

Em face do exposto, a rotina atual do The Hospital for Sick Children, de Londres, é baseada no uso de homoenxertos aórticos, ou pulmonares, preservados em meio antibiótico-nutriente, com pequeno intervalo de tempo de estoque, evitando-se o uso de extensão de Dacron.

\begin{abstract}
ALMEIDA, R. S.; WYSE, R.; DE LEVAL, M.; STARK, J. - The use of homograph and heterograph valves in extracardiac conduits. Rev. Bras. Cir. Cardiovasc., 3(2): 101-108, 1988.

ABSTRACT: The concept of using extracardiac conduits, to establish an outflow tract between the right ventricle and the pulmonary artery was developed on the sixties. Between 1971 and 1986, 335 patients received extracardiac conduits for the right heart, at The Hospital for Sick Children, London; 176 were antibiotic preserved aortic homografts (Hancock, Ross, Carpentier-Edwards, Ionescu-Shiley) and 19 non-valved tubes. These conduits were used for the repair of complex congenital heart defects. The mean age of these groups was $6.34 \pm 4.6$ years and the mean weight $17.8 \pm 10.8 \mathrm{~kg}$. The internal diameter of the conduits varied from 8 to $30 \mathrm{~mm}$. The hospital mortality was $29.2 \%$ and long-term follow-up of the survivals had a maximum period of 14,39 years. Sixty patients $(17.9 \%)$ were submited to 60 reoperations, being only $40 \%$ conduit related. The actuarial survival curve of freedom from obstruction was significant when analyzed the homografts, without Dacron, versus each group of heterografts $(p<0.005)$. The more important risk factors were the number of postoperative complications, for late deaths; the severity of associated lesions for reoperation, the date of surgery for the replacement of the extracardiac conduit, and the date of surgery and severity of associated lesions for the obstruction of the extracardiac conduits. We conclude that on long-term the use of extracardiac valved conduits has good results specially when using antibiotic preserved aortic homografts and therefore they are our first choice in the reconstruction of the pulmonary ventricle outflow tract.
\end{abstract}

DESCRIPTORS: extracardiac conduits, surgery; heart valves, surgery; heart valves prostheses, surgery.

\title{
REFERÊNCIAS BIBLIOGRÁFICAS
}

1 ALMEIDA, R. S.; WYSE, R. K. H.; ELLIOT, M. J.; DE LEVAL, M. R.; STARK, J. - Long-term experience with "fresh" antibiotic sterelized aortic homografts as extracardiac conduits (No prelo).
2 ALMEIDA, R. S.; WYSE, R. K. H.; ELLIOT, M. J.; DE LEVAL, M. R.; STARK, J. - Long-term results of homograft valves in extracardiac valved conduits in relation to the method of preparation (No prelo). 
ALMEIDA, R. S.; WYSE, R.; DELEVAL, M.; STARK, J. - Utilização de valvas homólogas e heterólogas em condutos extracardíacos. Rev. Bras. Cir. Cardiovasc., 3(2): 101-108, 1988.

3 BAILEY, W. W.; KIRKLIN, J. W.; BARGERON Jr., L. M.; PACIFICO, A. D.; KOUCHOUKOS, N. T. - Late results with synthetic valved external conduits from venous ventricle to pulmonary arteries. Circulation, 56 (Supl. 2): $73-79,1977$.

4. BULL, C.; MaCARTNEY, F. J.; HORVATH, P.; AL.MEIDA R.; MERRIL, W.; DOUGLAS, J.; TAYLOR, J. F. N.; DE LEVAL, M. R.; STARK J. - Evaluation of long-term results of homograft and heterograft valves in extracardiac conduits. J. Thorac. Cardiovasc. Surg., 94 (1): 12-19, 1987.

5 CASTAÑEDA, A. R. \& NORWOOD, W. I. - Valved conduits: a panacea for complex congenital heart defects? In: COHN, L. H. \& GALLUCCI, V. eds. Cardiac bioprosthesis. New York, Yorke Medical Books, 1982. p. 205-216.

6 DOTY, D. B.; MARVIN. W. J.; LAVER, R. M. - Modified Fontan procedure: methods to achieve direct anastomosis of right atrium to pulmonary artery. J. Thorac. Cardiovasc. Surg., 81 (3): 470:475, 1981.

7 EBERT, P. A.; TURLEY, K.; STANGER, P.; HOFFMAN, J. I. E.; HEYMANN, M. A.; RUDOLPH, A. M. - Surgical treatment of truncus arteriosus in the frist 6 months of life. Ann. Surg., 200 (4): 451-456, 1984.

8 FONTAN, F.; CHOUSSAT, A.; DEVILLE, C.; DOUTREMEPUICH, C.; COUPILLAUD, J.; VOSA, C. - Aortic valve homografts in the surgical treatment of complex cardiac malformations. J. Thorac. Cardiovasc. Surg., 87 (5): 649-657, 1984.

9 JACOBS, T.; DE LEVAL, M.; STARK, J. - False aneurysm of right ventricle after Rastelli operation for transposition of the great arteries, ventricular septal defect and pulmonary stenosis. J. Thorac. Cardiovasc. Surg., 67 (4): 543-546, 1974.

10 JONAS, R. A.; FREED, M. D.; MAYER Jr., J. E.; CASTANEDA, A. R. - Long-term follow-up of patients with synthetic, right heart conduits. Circulation, 72 (Supl. 2): $77-83,1985$.

11 KAY, P. H. \& ROSS, D. N. - Fifteen years experience with aortic homografts: the conduit of choice for right ventricular outflow tract reconstruction. Ann. Thorac. Surg., 40 (4) : 360-364, 1985.

12 LAYTON, C. A.; MC DONALD, A.; Mc DONALD, L.; TOWERS, M.; WEAVER, J.; YACOUB, M. - The syndrome of absent pulmonary valve: total correction with aortic valvular homografts. J Thorac. Cardiovasc. Surg., 63 (5): 800-808, 1972.

13 LECOMPT, Y.; NEVEAUX, J. Y.; LECA, F.; ZANINI, L.; TU, T. V.; DUBOYS, Y.; JARREAU, M. M. - Reconstruction of the pulmonary outflow tract without prosthetic conduit. J. Thorac. Cardiovasc. Surg., 84 (5): 727-733, 1982.

14 LIVI, V.; ABDULA, A.; PARKER, R.; OLSEN, E. J.; ROSS, D. N. - Viability and morphology of aortic and pulmonary homografts: a comparative study. J. Thorac. Cardiovasc. Surg., 93 (5): 755-760, 1987.
15 McGOON, D. C.; DANIELSON, G. K.: PUGA, F. J.; RITTER, D. G.; MAIR, D. D.: ILSTRUP, D. M. - Late results after extracardiac conduit repair for congenital cardiac defects. Am. J. Cardiol., 49(5): 1741-1749, 1982.

16 MCGOON, D. C.; DANIELSON, G. K.; SCHAFF, H. V.; PUGA, F. J.; RITTER, D, G.; MAIR, D. D.; ILSTRUP, D. M.; EDWARDS, W. D. - Factors influencing late results of extracardiac conduit repair for congenital cardiac defects. In: COHN, L H. \& GALLUCCI, V. eds. Cardiac bioprosthesis. New York, Yorke Medical Books, 1982. p. 217-230.

17 MELDRUN-HANNA, W.; CARTHILL, T.; JOHNSON, D. CELERMAJER, J.; HAWKER, R. - Late results of right ventricular outflow tract reconstruction using a valved extracardiac conduit. In: BODNAR, E \& YACOUB, M. eds. Biologic and bioprosthetic valves. New York, Yorke Medical Books, 1986. p. 305-315.

18 MERIN, G. \& McGOON, D. C. - Reoperation after insertion of aortic homograft as a right ventricular outflow tract. Ann. Thorac. Surg., 16 (2): 122-126, 1973.

19 RASTELLI, G. C.; ONGLEY, P. A.; DAVIS, G. D.; KIRKLIN, J. W. - Surgical repair for pulmonary valve atresia with coronary-pulmonary artery fistula: report of a case. Mayo Clin. Proc., 40: 512-527, 1965.

ROBBARD, S. \& WAGNER, D. - By-passing the right ventricle. Proc. Soc. Exp. Biol. Med., 71:69-70, 1949.

21 ROSS, D. N. \& SOMERVILLE, J. - Correction of pulmonary atresia with a homograft aortic valve. Lancet, 2 (7479): 1446-1447, 1966.

22 SARAVALLI, O. A.; SOMERVILLE, J.; JEFFERSON, $\mathrm{K}$. E. - Calcification of the aortic homografts used for reconstruction of the right ventricular outflow tract. J. Thorac. Cardiovasc. Surg., 80 (6): 909-920, 1980.

23 SHABBO, F. P.; WAIN, W. H.; ROSS, D. N. - Right ventricular outflow tract reconstruction with aortic homograft conduit: analysis of the long-term results. J. Thorac. Cardiovasc. Surg., 28 (1): 21-25, 1980.

24 STEWART, S.; MANNING, J.; ALEXSON, C.; HARRIS, P. - The Hancock external valved reconstruction with aortic homograft conduit: a dichotomy between late clinical results and late cardiac catheterization findings. J. Thorac. Cardiovasc. Surg., 86(4): 562-569, 1983.

25 WEST, P. N.; HARTMANN Jr., A. F.; WELDON, C. S. - Long-term function of aortic homografts as the right ventricular outflow tract. Circulation, 56 (Supl. 2): 66-72, 1977.

\section{Discussão}

\section{DR. PAULO P. PAULISTA \\ São Paulo, SP}

Primeiramente, meus melhores cumprimentos ao colega Rui de Almeida, pela excelência da apresentação 
e do material mostrado. No Instituto Dante Pazzanese de Cardiologia, em Sāo Paulo, no período de 12 anos, (slide) entre 1976 e 1988 , foram operados 62 pacientes, nos quais se fez uso de conduto extracardiaco, para tratamento de cardiopatia congênita. Esses casos eram, em sua maioria, do sexo masculino (39) e a idade variou de 38 dias a 30 anos, com média de 55 meses. O peso oscilou entre 3,4 a 57 quilos, com média de 16 quilos. As patologias presentes (slide) foram as seguintes: atresia pulmonar, com 17 casos e 5 óbitos $(29,4 \%)$; transposição das grandes artérias com CIV e estenose pulmonar, 17 casos, com 2 óbitos (11,7\%); truncus arteriosus, com 17 casos e 7 óbitos $(41,1 \%)$; dupla via de saída de ventrículo direito com estenose pulmonar, 7 casos, com 2 óbitos $(28,5 \%)$ e 4 casos de tétrade de Fallot, com 1 óbito (25,0\%), perfazendo o total de 62 pacientes operados, e 17 óbitos na fase hospitalar, com $27,4 \%$ de mortalidade global. Como se pode verificar, o grupo de maior mortalidade, entre nós, foi o de truncus, com $41 \%$, como ocorre, freqüentemente, na literatura internacional, neste tipo de operação. Dos 62 pacientes, (slide) em 37 utilizou-se um conduto valvulado, com 11 óbitos $(29,7 \%)$ e, em 25 , o conduto era avalvulado, com 6 óbitos $(24,0 \%)$. O conduto empregado foi o tubo de Dacron de média, ou de baixa porosidade, ou o tubo construído com material biológico (dura-máter, ou pericárdio bovino). A válvula, quando presente, foi mecânica, ou biológica e, entre estas, de dura-máter, pericárdio bovino, presentes no comércio, ou construídas no momento de utilização, pelo cirurgiāo, com 1 ou 3 folhetos. Fato interessante é que, na mortalidade hospitalar, a presença, ou a ausência, da válvula não modificou, apreciavelmente, o resultado cirúrgico, mostrando que a escolha por um outro tipo de conduto, de acordo com o caso presente, foi acertada. Neste grupo, ocorreram 2 reoperaçōes, em 1 caso de truncus e, em outro, de L-transposição com CIV e estenose pulmonar. Em ambos, o resultado da reoperação foi muito bom. Finalmente, neste último (slide), aprecia-se que, em 14 pacientes com cirurgia paliativa prévia (predominantemente BlalockTaussig clássico), ocorreram apenas 2 óbitos, com $14,2 \%$ de mortalidade. Entre os 48 doentes sem cirurgia prévia, ocorreram 15 óbitos, com $31,2 \%$ de mortalidade no hospital. Embora não se possa afirmar, houve diferença significativa entre os 2 grupos, parecendo que a operação prévia, melhorando as condiçōes do paciente e permitindo que a intervenção definitiva seja feita com maior idade e peso, com emprego de condutos de maior diâmetro, traz resultados imediatos melhores. Possivelmente, a necessidade de reoperações por estenose de conduto também sejam proteladas por mais tempo. A conduta de nosso Serviço, sempre que possivel, tem sido a de fazer cirurgia de shunt prévia, se indicada, para empregar o conduto no paciente com idade em torno de 4 anos. A bandagem pulmonar não tem tido indicação, especialmente nos casos de truncus, quando a cirurgia definitiva é a primeira escolha, apesar da necessidade de emprego de tubos de menor calibre. O fato de realizar-se essa cirurgia em idade precoce, neste grupo, deve levar à maior mortalidade hospitalar. Os 7 pacientes com truncus, que faleceram, tinham, todos eles, idade inferior a 6 meses. Eram estas as consideraçōes que desejávamos fazer e queremos, mais uma vez, parabenizar o Dr. Rui, além de agradecer à Comissão Organizadora a lembrança de nosso nome como comentador.

\section{DR. RUI S. DE ALMEIDA (Encerrando)}

Eu gostaria de agradecer ao Dr. Paulo Paulista, pelos comentários. O material apresentado pelo colega mostra, sem dúvida, a precisa indicaçāo e a alta técnica cirúrgica de seu grupo, pois seus resultados são idênticos aos melhores da literatura internacional, embora a amostragem de pacientes seja pequena. No entanto, o nosso estudo concentrou-se mais nos resultados a longo prazo destes pacientes, o que nos dá uma melhor idéia da sobrevida de cada tipo de conduto extracardíaco. A conclusão de nosso estudo é que, a longo prazo, os homoenxertos sem extensão têm melhor sobrevida, livre de obstrução, que todos os outros condutos, incluindo um grupo semelhante em número, que é o grupo de heteroenxertos tipo Hancock. Por este motivo, o homoenxerto aórtico preservado em meio anbitiótico-nutriente continua sendo o nosso conduto extracardiaco preferido. 